\title{
PBW DEFORMATIONS OF A FOMIN-KIRILLOV ALGEBRA AND OTHER EXAMPLES
}

\author{
I. HECKENBERGER AND L. VENDRAMIN
}

\begin{abstract}
We begin the study of PBW deformations of graded algebras relevant to the theory of Hopf algebras. One of our examples is the Fomin-Kirillov algebra $\mathcal{E}_{3}$. Another one appeared in a paper of García Iglesias and Vay. As a consequence of our methods, we determine when the deformations are semisimple and we are able to produce PBW bases and polynomial identities for these deformations.

Keywords: Clifford algebra, Fomin-Kirillov algebra, Hopf algebra, Nichols algebra, PBW deformation, polynomial identity.

Corresponding author: Leandro Vendramin
\end{abstract}

\section{INTRODUCTION}

Deformations of several algebraic structures have been of great interest in the last years. Such deformations include group algebras, Lie algebras, Weyl algebras, rational Cherednik algebras, Hecke algebras and generalizations. A deformation of a graded algebra $A$ given by generators $a_{1}, \ldots, a_{n}$ and homogeneous relations $r_{1}, \ldots, r_{m}$ is an algebra $D$ given by generators $a_{1}, \ldots, a_{n}$ and relations $r_{1}+t_{1}, \ldots, r_{m}+t_{m}$, where each $t_{j}$ is a possibly non-homogeneous element of degree less than the degree of $r_{j}$.

The classical Poincaré-Birkhoff-Witt Theorem for Lie algebras motivates the study of a particular family of deformations. A PBW deformation of a graded algebra $A$ is a deformation $D$ of $A$ such that the associated graded algebra of $D$ is isomorphic to $A$. PBW deformations have been considered in many different contexts. In the case of quadratic algebras, these deformations have been recently studied in [7, 9, 14, 26]. For $N$-homogeneous algebras they have been studied in [12, 5]. In the context of Hopf algebras and their actions, a related class of algebras was studied in [30] and in [29]. PBW deformations satisfying additional properties also appear in several papers where the classification of finite-dimensional pointed Hopf algebras is considered, see for example [15].

Nichols algebras over non-abelian groups form a particularly interesting family of graded algebras where not very much is known. In general, they are not $N$-Koszul and are not even generated by homogeneous relations of

1991 Mathematics Subject Classification. Primary 16T05; Secondary 20F55.

The second-named author is partially supported by PICT-2014-1376, MATH-AmSud 17MATH-01, ICTP, ERC advanced grant 320974 and the Alexander von Humboldt Foundation. 
constant degree. Nevertheless they have remarkable Hilbert series which indicates a rich internal structure. We refer to [3] for an introduction to the theory of Nichols algebras. At this place we do not want to go into details, since we will only use their known presentations as algebras by generators and relations. Many of the crucial results on Nichols algebras over non-abelian groups can only be recovered by extensive Gröbner basis calculations. Nevertheless, these algebras are very important in particular since they appear as an essential tool in the classification of pointed Hopf algebras with non-abelian coradical [1, in combinatorics [4, 6, 13, 19, 22] and in mathematical physics [18, 20, 21, 25].

In this paper we begin the study of PBW deformations of some finitedimensional Nichols algebras over non-abelian groups. Our long-term objective is to understand the structure of finite-dimensional Nichols algebras and Fomin-Kirillov algebras by means of PBW deformations. For that purpose we concentrate first on two small examples: 1) The Fomin-Kirillov algebra $\mathcal{E}_{3}$, and 2) the Nichols algebra associated with the vertices of the tetrahedron and constant cocycle -1 .

Some particular deformations of the Fomin-Kirillov algebra $\mathcal{E}_{3}$ have been considered in [21, 20] and in [27]. The cohomology of $\mathcal{E}_{3}$ has been recently computed in [8].

We compute all PBW deformations of these Nichols algebras. It turns out that the moduli space of PBW deformations is an affine space of very small dimension. Moreover, generically the deformations are semisimple and the non-semisimple locus is determined. Our result has some applications: a) we get a PBW basis of the algebras; b) we produce non-trivial polynomial identities for our Nichols algebras that were not known before. For example, as a corollary we prove that the Fomin-Kirillov algebra $\mathcal{E}_{3}$ satisfies the Hall identity

$$
\left[[x, y]^{2}, z\right]=0 .
$$

The PBW deformations we find have also been obtained by García Iglesias and Vay [25]. These algebras are useful to obtain the classification of some finite-dimensional pointed Hopf algebras with non-abelian coradical. We provide a simplification in the presentation of the 72-dimensional example.

The paper is organized as follows. In Section 1 we define our algebras as certain PBW deformations of Nichols algebras compatible with the group action and we prove some basic properties. Then we recall a basic result on the simplicity of Clifford algebras that will be useful to study our examples. In Section 2 we study PBW deformations of the Fomin-Kirillov algebra $\mathcal{E}_{3}$. In Theorem 2.11 we precisely determine when the deformations of $\mathcal{E}_{3}$ are semisimple; it is remarkable that in the cases where the deformation is not semisimple one finds either the preprojective algebra of type $A_{2}$ or the coinvariant algebra appearing in Schubert calculus [4. In Theorem 2.12 a PBW basis is constructed and PBW deformations of $\mathcal{E}_{3}$ are classified in Theorem 2.15. In Section 4PBW deformations of the 72-dimensional Nichols 
algebra associated with the vertices of the tetrahedron are studied. Using Ore extensions we produce a PBW basis for the deformation of this Nichols algebra, see Proposition 4.8, Theorems 4.9 and 4.13. In Theorem 4.11 we determine when these deformations are semisimple. Most of the results on this deformation are based on calculations related to an intermediate algebra studied in Section 3.

We end the introduction by formulating three problems.

Problem 1. Classify the PBW deformations of the known finite-dimensional Nichols algebras over groups. Decide when these algebras are semisimple.

Problem 2. Classify the PBW deformations of the Fomin-Kirillov algebras and their subalgebras appearing in [6]. Decide when these deformations are semisimple.

Problem 3. Study the representation theory of the PBW deformations of the algebras in the first two problems.

Acknowledgments. We thank Pavel Etingof for pointing out the references [10, 11].

\section{Preliminaries}

Throughout this paper we assume that $\mathbb{K}$ is an algebraically closed field of characteristic $\neq 2$.

1.1. Deformations. Let $\mathcal{C}$ be a monoidal category. (In our case, $\mathcal{C}$ will be the category of $\mathbb{K} G$-modules for some field $\mathbb{K}$ and some finite group $G$.)

Definition 1.1. Let $A$ be an $\mathbb{N}_{0}$-graded algebra in $\mathcal{C}$. A PBW deformation of $A$ in $\mathcal{C}$ is an $\mathbb{N}_{0}$-filtered algebra $D_{A}$ in $\mathcal{C}$ such that gr $D_{A} \cong A$.

In our study we usually meet particular families, which will be defined here.

Definition 1.2. Let $A$ be an $\mathbb{N}_{0}$-graded algebra over the field $\mathbb{K}$ and let $s \geq 1$ be an integer. We say that a family $(A(\lambda))_{\lambda \in \mathbb{K}^{s}}$ is an affine family of deformations of $A$ if there are a family $\left(a_{i}\right)_{i \in I}$ of homogeneous generators of $A, a$ family $\left(r_{j}\right)_{j \in J}$ of homogeneous relations of $A$, a basis $\left(b_{l}\right)_{l \in L}$ of $A$ consisting of homogeneous elements, and a family $\left(c_{j l}\right)_{j \in J, l \in L} \in \mathbb{K}\left[t_{1}, \ldots, t_{s}\right]^{J \times L}$ such that $A(0)=A$ and for any $\lambda \in \mathbb{K}^{s}$,

$$
A(\lambda)=\mathbb{K}\left\langle a_{i} \mid i \in I\right\rangle /\left(r_{j}+\sum_{l \in L} c_{j l}(\lambda) b_{l} \mid j \in J\right),
$$

and $c_{j l}=0$ whenever $\operatorname{deg}\left(b_{l}\right) \geq \operatorname{deg}\left(r_{j}\right)$.

Clearly, being an affine family of deformations of an $\mathbb{N}_{0}$-graded algebra does not depend on the choice of homogeneous generators and defining relations. 
The following proposition is well-known and the proof is elementary. Similar arguments have been used by Etingof and Rains for example in [11, §2.2] and [10, Theorem 6.1]. We refer to [24 for an introduction to the theory of non-commutative Gröber basis.

Proposition 1.3. Let $A$ be an $\mathbb{N}_{0}$-graded finite-dimensional algebra over $\mathbb{K}$. Let $s, d \in \mathbb{N}$ and let $(A(\lambda))_{\lambda \in \mathbb{K}^{s}}$ be an affine family of deformations of $A$ such that $\operatorname{dim} A(\lambda) \leq d$ for all $\lambda \in \mathbb{K}^{s}$. Assume that $\operatorname{dim} A(\lambda)=d$ for all $\lambda$ in a Zariski dense subset of $\mathbb{K}^{s}$. Then $A(\lambda)$ is a PBW deformation of $A$ for all $\lambda \in \mathbb{K}^{s}$.

Proof. Choose a family $\left(a_{i}\right)_{i \in I}$ of homogeneous generators of $A$, a family $\left(r_{j}\right)_{j \in J}$ of homogeneous relations of $A$, a basis $\left(b_{l}\right)_{l \in L}$ of $A$ consisting of homogeneous elements, and a family $\left(c_{j l}\right)_{j \in J, l \in L} \in \mathbb{K}\left[t_{1}, \ldots, t_{s}\right]^{J \times L}$, such that $A(0)=A$ and for any $\lambda \in \mathbb{K}^{s}$,

$$
A(\lambda)=\mathbb{K}\left\langle a_{i} \mid i \in I\right\rangle /\left(r_{j}+\sum_{l \in L} c_{j l}(\lambda) b_{l} \mid j \in J\right),
$$

and $c_{j l}=0$ whenever $\operatorname{deg}\left(b_{l}\right) \geq \operatorname{deg}\left(r_{j}\right)$. We may assume that $\left(r_{j}\right)_{j \in J}$ is a Gröbner basis of $A$. Then, by assumption,

$$
\left(r_{j}+\sum_{l \in L} c_{j l}(\lambda) b_{l}\right)_{j \in J}
$$

is a Gröbner basis of $A(\lambda)$ for any $\lambda$ in a Zariski dense subset $\Lambda$ of $\mathbb{K}^{s}$. Let $p$ be an $S$-polynomial. Then $p$ reduces with respect to the family (1.1) to a polynomial $\sum_{l \in L} c_{l}(\lambda) b_{l}$, where $c_{l} \in \mathbb{K}\left[t_{1}, \ldots, t_{s}\right]$ and each restriction $c_{l} \mid \lambda$ is zero. It follows that $c_{l}=0$ for all $l \in L$, and hence for any $\lambda \in \mathbb{K}^{s}$, (1.1) is a Gröbner basis of $A(\lambda)$. This implies the claim.

1.2. Clifford algebras. Let $V$ be a finite-dimensional vector space with basis $v_{1}, \ldots, v_{n}$ and let $q$ be a quadratic form on $V$. The pair $(V, q)$ is called a quadratic space. The Clifford algebra $C(V, q)$ is the algebra given by generators $v_{1}, \ldots, v_{n}$ and relations

$$
v_{i}^{2}=q\left(v_{i}\right), \quad v_{j} v_{k}+v_{k} v_{j}=2 q_{j k}
$$

for $1 \leq i, j, k \leq n$ with $j<k$, where $q_{j k}=\frac{1}{2}\left(q\left(v_{j}+v_{k}\right)-q\left(v_{j}\right)-q\left(v_{k}\right)\right)$. It is known that $\operatorname{dim} C(V, q)=2^{n}$, see for example [28, §9.2, Corollary 2.7].

Recall that the radical of a symmetric bilinear form $B$ on a vector space $V$ is the subspace of elements $v \in V$ with $B(v, w)=0$ for all $w \in V$.

Theorem 1.4. Let $(V, q)$ be a quadratic space.

(1) The radical of $C(V, q)$ is generated by the radical of the symmetric bilinear form $B_{q}$ associated with $q$.

(2) If $\operatorname{dim} V$ is even and $q$ is nondegenerate, then $C(V, q)$ is simple.

(3) If $\operatorname{dim} V$ is odd and $q$ is nondegenerate, then $C(V, q)$ is the product of two simple ideals of dimension $2^{\operatorname{dim} V-1}$ each. 
Proof. The claims (2) and (3) follow from [28, §9.2, Theorem 2.10]. Regarding (1), note that for any element $v$ in the radical $R$ of $B_{q}$, the left ideal generated by $v$ is a nilpotent two-sided ideal of $C(V, q)$. Hence

$$
C(V, q) R \subseteq \operatorname{Rad} C(V, q) .
$$

Let $q^{\prime}$ be the quadratic form on $V / R$ induced by $q$. Then $C\left(V / R, q^{\prime}\right)$ is semisimple by (2) and (3) and $C(V, q) / C(V, q) R \simeq C\left(V / R, q^{\prime}\right)$. Hence $\operatorname{Rad} C(V, q)=C(V, q) R$.

\section{The Fomin-Kirillov algebra $\mathcal{E}_{3}$}

The Fomin-Kirillov algebra $\mathcal{E}_{3}$ is defined by generators $a, b, c$ and relations

$$
\begin{aligned}
& a^{2}=b^{2}=c^{2}=0, \\
& c a+b c+a b=c b+b a+a c=0 .
\end{aligned}
$$

It is known that $\operatorname{dim} \mathcal{E}_{3}=12$. A basis is given by

$$
1, a, b, c, a b, a c, b a, b c, a b a, a b c, b a c, a b a c .
$$

We put this algebra into a different context without using this information.

Remark 2.1. It is known that $\mathcal{E}_{3}$ together with an appropriate comultiplication, counit and antipode is a Nichols algebra. This was first proved by Milinski and Schneider [23. The primitive elements of this Nichols algebra are spanned by $a, b$ and $c$.

The Fomin-Kirillov algebra $\mathcal{E}_{3}$ first appeared in 13 to provide a combinatorial tool to study the structure constants of Schubert polynomials, and independently in the paper [23] of Milinski and Schneider, where pointed Hopf algebras with non-abelian coradical were studied. It also appeared in the work of Majid and Raineri [21], where applications to physics where considered. The cohomology of $\mathcal{E}_{3}$ was computed by Ştefan and Vay in [8].

Definition 2.2. For any $\alpha_{1}, \alpha_{2} \in \mathbb{K}$ let $\mathcal{D}_{3}\left(\alpha_{1}, \alpha_{2}\right)$ be the deformation of $\mathcal{E}_{3}$ given by generators $a, b, c$ and relations

$$
\begin{aligned}
& a^{2}-\alpha_{1}=b^{2}-\alpha_{1}=c^{2}-\alpha_{1}=0, \\
& c a+b c+a b-\alpha_{2}=c b+b a+a c-\alpha_{2}=0 .
\end{aligned}
$$

Note that the algebras $\mathcal{D}_{3}\left(\alpha_{1}, \alpha_{2}\right)$ are deformations of $\mathcal{E}_{3}$ by definition. However, it is not a priori clear that they are PBW deformations.

For the rest of the section let $\alpha_{1}, \alpha_{2} \in \mathbb{K}$.

Remark 2.3. A direct calculation shows that a Gröbner basis for the defining ideal of $\mathcal{D}_{3}\left(\alpha_{1}, \alpha_{2}\right)$ is given by

$$
\begin{array}{lll}
a^{2}-\alpha_{1}=0, & b^{2}-\alpha_{1}=0, & c a+b c+a b-\alpha_{2}=0, \\
c b+b a+a c-\alpha_{2}=0, & c^{2}-\alpha_{1}=0, & b a b-a b a-\alpha_{2} b+\alpha_{2} a=0 .
\end{array}
$$

We will not use this Gröbner basis for our arguments. 
Remark 2.4. The algebra $\mathcal{D}_{3}\left(\alpha_{1}, \alpha_{2}\right)$ is an $\mathbb{S}_{3}$-module algebra, where

$$
\begin{aligned}
(12) \cdot a=-b, & (12) \cdot b=-a, & (12) \cdot c=-c, \\
(23) \cdot a=-a, & (23) \cdot b=-c, & (23) \cdot c=-b .
\end{aligned}
$$

Lemma 2.5. Let $u=a-b, v=b-c$ and $w=c-a$ in $\mathcal{D}_{3}\left(\alpha_{1}, \alpha_{2}\right)$. Then

$$
\begin{aligned}
& u a=-b u, \quad u b=-a u, \quad u c=(c-a-b) u, \\
& u v=v u, \quad u w=w u, \quad v w=w v .
\end{aligned}
$$

\section{Moreover}

$$
u v+v w+u w=\alpha_{2}-3 \alpha_{1}, \quad u^{3}=\left(3 \alpha_{1}-\alpha_{2}\right) u, \quad u^{2} v+u v^{2}=0, \quad u v w=0 .
$$

Proof. The first formula is obtained as follows:

$$
u a=(a-b) a=a^{2}-b a=b^{2}-b a=b(b-a)=-b u .
$$

By acting with $(12) \in \mathbb{S}_{3}$ we obtain that $u b=-a u$. Now

$$
\begin{aligned}
(c-a-b) u & =(c-a-b)(a-b)=c a-c b-\alpha_{1}+a b-b a+\alpha_{1} \\
& =c a+a b-(c b+b a)=-b c+\alpha_{2}+a c-\alpha_{2}=(a-b) c=u c .
\end{aligned}
$$

We conclude that $u v=v u$ and $u w=w u$. By acting with (23) on $u v=v u$ we obtain that $v w=w v$.

From the definitions of $u, v$ and $w$ it follows that

$$
u v+v w+u w=v w-u^{2}=\alpha_{2}-3 \alpha_{1} .
$$

From $c b+b a+a c-\alpha_{2}=0$ one obtains that

$$
b c b+\alpha_{1} a+b a c-\alpha_{2} b=0=\alpha_{1} c+b a b+a c b-\alpha_{2} b .
$$

Hence a direct calculations shows that

$$
u^{2} v+u v^{2}=\alpha_{1} a-\alpha_{1} c-b a b+b a c-a c b+b c b=-\alpha_{2} b+\alpha_{2} b=0 .
$$

Moreover

$$
u v w=u v(-u-v)=-u^{2} v-u v^{2}=0
$$

Finally

$$
u^{3}=-u^{2}(v+w)=-u(u v+u w+v w)+u v w=\left(3 \alpha_{1}-\alpha_{2}\right) u .
$$

This completes the proof.

Lemma 2.6. Assume that $3 \alpha_{1}-\alpha_{2} \neq 0$. Then the elements

$$
\begin{aligned}
e_{1} & =\frac{1}{3 \alpha_{1}-\alpha_{2}}\left((b+c)^{2}-\left(\alpha_{1}+\alpha_{2}\right)\right)=\frac{1}{3 \alpha_{1}-\alpha_{2}}(c-a)(b-a), \\
e_{2} & =\frac{1}{3 \alpha_{1}-\alpha_{2}}\left((a+c)^{2}-\left(\alpha_{1}+\alpha_{2}\right)\right)=\frac{1}{3 \alpha_{1}-\alpha_{2}}(c-b)(a-b), \\
e_{3} & =\frac{1}{3 \alpha_{1}-\alpha_{2}}\left((a+b)^{2}-\left(\alpha_{1}+\alpha_{2}\right)\right)=\frac{1}{3 \alpha_{1}-\alpha_{2}}(a-c)(b-c),
\end{aligned}
$$

form a set of orthogonal central idempotents of $\mathcal{D}_{3}\left(\alpha_{1}, \alpha_{2}\right)$. 
Proof. Using the first formulas for $e_{1}, e_{2}$ and $e_{3}$, we conclude from the definition of $\mathcal{D}_{3}\left(\alpha_{1}, \alpha_{2}\right)$ that $1=e_{1}+e_{2}+e_{3}$. A direct calculation using Lemma 2.5 shows that $e_{2}$ is an idempotent that commutes with $a, b$ and $c$. Then $e_{1}=(12) \cdot e_{2}$ and $e_{3}=(23) \cdot e_{2}$ are central idempotents. From Lemma 2.5 it now follows that $e_{1}, e_{2}$ and $e_{3}$ are orthogonal.

Lemma 2.7. Assume that $3 \alpha_{1}-\alpha_{2} \neq 0$. Let $V$ be a vector space with basis $x_{1}, x_{2}$ and let $q: V \rightarrow \mathbb{K}$ be the quadratic form given by

$$
q\left(\lambda_{1} x_{1}+\lambda_{2} x_{2}\right)=\alpha_{1} \lambda_{1}^{2}+\left(\alpha_{2}-\alpha_{1}\right) \lambda_{1} \lambda_{2}+\alpha_{1} \lambda_{2}^{2} .
$$

Then $e_{3} \mathcal{D}_{3}\left(\alpha_{1}, \alpha_{2}\right) \simeq C(V, q)$.

Proof. Lemma 2.5implies that $e_{3}(a-b)=0$. Then the algebra $e_{3} \mathcal{D}_{3}\left(\alpha_{1}, \alpha_{2}\right)$ with unit $e_{3}$ is given by generators $e_{3} a, e_{3} c$ and relations

$$
\begin{gathered}
\left(e_{3} a\right)^{2}=\left(e_{3} c\right)^{2}=\alpha_{1} e_{3}, \\
e_{3} c e_{3} a+e_{3} a e_{3} c=\left(\alpha_{2}-\alpha_{1}\right) e_{3}, \\
\left(e_{3} a-e_{3} c\right)^{2}=\left(3 \alpha_{1}-\alpha_{2}\right) e_{3} .
\end{gathered}
$$

Since (2.3) follows from the other equalities, the lemma holds.

Remark 2.8. The symmetric bilinear form $B$ of the quadratic form $q$ in Lemma 2.7 satisfies

$$
B\left(x_{1}, x_{1}\right)=B\left(x_{2}, x_{2}\right)=\alpha_{1}, \quad B\left(x_{1}, x_{2}\right)=\frac{1}{2}\left(\alpha_{2}-\alpha_{1}\right) .
$$

It follows that $B$ is degenerate if and only if $\alpha_{1}^{2}-\frac{1}{4}\left(\alpha_{2}-\alpha_{1}\right)^{2}=0$, that is, $\left(3 \alpha_{1}-\alpha_{2}\right)\left(\alpha_{1}+\alpha_{2}\right)=0$.

Proposition 2.9. Assume that $\left(3 \alpha_{1}-\alpha_{2}\right)\left(\alpha_{1}+\alpha_{2}\right) \neq 0$. Then $e_{1} \mathcal{D}_{3}\left(\alpha_{1}, \alpha_{2}\right)$, $e_{2} \mathcal{D}_{3}\left(\alpha_{1}, \alpha_{2}\right)$ and $e_{3} \mathcal{D}_{3}\left(\alpha_{1}, \alpha_{2}\right)$ are simple algebras isomorphic to $\mathbb{K}^{2 \times 2}$.

Proof. Using the group action one proves that these algebras are isomorphic. So it suffices to prove that $e_{3} \mathcal{D}_{3}\left(\alpha_{1}, \alpha_{2}\right)$ is a simple algebra. By Lemma 2.7 . the latter algebra is isomorphic to a Clifford algebra. Thus the simplicity of $e_{3} \mathcal{D}_{3}\left(\alpha_{1}, \alpha_{2}\right)$ follows from and Theorem 1.4 and Remark 2.8

Next we discuss the deformation $\mathcal{D}_{3}\left(\alpha_{1}, 3 \alpha_{1}\right)$.

Proposition 2.10. Let $\mathcal{L}$ denote the left ideal of $\mathcal{D}_{3}\left(\alpha_{1}, 3 \alpha_{1}\right)$ generated by $a-b$ and $b-c$. Then $\mathcal{L}$ is a two-sided nilpotent ideal of $\mathcal{D}_{3}\left(\alpha_{1}, 3 \alpha_{1}\right)$. The quotient algebra $\mathcal{D}_{3}\left(\alpha_{1}, 3 \alpha_{1}\right) / \mathcal{L}$ has dimension 2 and is isomorphic to the algebra $\mathbb{K}[a] /\left(a^{2}-\alpha_{1}\right)$.

Proof. Let $u=a-b$ and $v=b-c$. Lemma 2.5 implies that $\mathcal{L}$ is a two-sided ideal. Moreover, $u^{3}=0$, and by acting with the transposition (13) we also obtain that $v^{3}=0$. Since $u v=v u$, it follows that $\mathcal{L}$ is nilpotent.

Adding $u$ and $v$ to the defining ideal of $\mathcal{D}_{3}\left(\alpha_{1}, 3 \alpha_{1}\right)$, one obtains the ideal

$$
\left(b-a, c-a, a^{2}-\alpha_{1}, 3 a^{2}-3 \alpha_{1}\right) .
$$

This implies the last claim. 
Now we prove the main theorems of this section.

Theorem 2.11. The algebra $\mathcal{D}_{3}\left(\alpha_{1}, \alpha_{2}\right)$ is semisimple if and only if

$$
\left(3 \alpha_{1}-\alpha_{2}\right)\left(\alpha_{1}+\alpha_{2}\right) \neq 0 .
$$

In this case $\mathcal{D}_{3}\left(\alpha_{1}, \alpha_{2}\right) \simeq\left(\mathbb{K}^{2 \times 2}\right)^{3}$.

Proof. By Proposition 2.10, $\mathcal{D}_{3}\left(\alpha_{1}, 3 \alpha_{1}\right)$ is not semisimple. So we may assume that $3 \alpha_{1}-\alpha_{2} \neq 0$. We decompose $\mathcal{D}_{3}\left(\alpha_{1}, \alpha_{2}\right)$ as

$$
\mathcal{D}_{3}\left(\alpha_{1}, \alpha_{2}\right) \simeq e_{1} \mathcal{D}_{3}\left(\alpha_{1}, \alpha_{2}\right) \oplus e_{2} \mathcal{D}_{3}\left(\alpha_{1}, \alpha_{2}\right) \oplus e_{3} \mathcal{D}_{3}\left(\alpha_{1}, \alpha_{2}\right),
$$

where $e_{1}, e_{2}, e_{3}$ are the central idempotents of Lemma 2.6. Now Proposition 2.9 implies that $\mathcal{D}_{3}\left(\alpha_{1}, \alpha_{2}\right)$ is semisimple and has dimension 12 if $\alpha_{1}+\alpha_{2} \neq 0$. In the case where $\alpha_{1}+\alpha_{2}=0$, the deformation $\mathcal{D}_{3}\left(\alpha_{1}, \alpha_{2}\right)$ is not semisimple by Lemma 2.7 and Theorem 1.4(1).

Theorem 2.12. The algebra $\mathcal{D}_{3}\left(\alpha_{1}, \alpha_{2}\right)$ is a $P B W$ deformation of $\mathcal{E}_{3}$ and

$$
(a-b)^{n_{1}} a^{n_{2}} c^{n_{3}}, \quad 0 \leq n_{1} \leq 2,0 \leq n_{2}, n_{3} \leq 1,
$$

is a basis of $\mathcal{D}_{3}\left(\alpha_{1}, \alpha_{2}\right)$.

Proof. Let $u=a-b$. Consider the lexicographic ordering on the words in the letters $u, a$ and $c$, induced by $u<a<c$. Definition 2.2 and Lemma 2.5 imply that $u^{3}=\left(3 \alpha_{1}-\alpha_{2}\right) u$ and

$$
\begin{aligned}
& a u=u^{2}-u a, \\
& c u=u^{2}-2 u a+u c, \\
& c a=\alpha_{2}-\alpha_{1}+u^{2}-u a+u c-a c .
\end{aligned}
$$

Hence the monomials $a u, c u, c a$ can be written as linear combinations of lexicographically smaller ordered monomials. Therefore (2.4) spans $\mathcal{D}_{3}\left(\alpha_{1}, \alpha_{2}\right)$. By Theorem 2.11 and Proposition 1.3. $\mathcal{D}_{3}\left(\alpha_{1}, \alpha_{2}\right)$ is a PBW deformation of $\mathcal{E}_{3}$ of dimension 12 for all $\alpha_{1}, \alpha_{2} \in \mathbb{K}$. Thus the 12 elements in (2.4) form a basis of $\mathcal{D}_{3}\left(\alpha_{1}, \alpha_{2}\right)$.

Remark 2.13. (1) In the proof of Theorem 2.12 we identified three quadratic relations (2.5) of $\mathcal{D}_{3}\left(\alpha_{1}, \alpha_{2}\right)$. Together with the relations $a^{2}=c^{2}=\alpha_{1}$ these form a set of defining relations. Indeed, $\mathcal{D}_{3}\left(\alpha_{1}, \alpha_{2}\right)$ is defined by three generators and five linearly independent quadratic relations for them. Using Gröbner basis calculations it is possible to check that for any $\alpha_{1}, \alpha_{2} \in \mathbb{K}$, none of these relations is superfluous.

(2) From the PBW basis of $\mathcal{E}_{3}=\mathcal{D}_{3}(0,0)$ in Theorem 2.12 one recovers quickly that the Hilbert series of $\mathcal{E}_{3}$ is the polynomial $(1+t)^{2}\left(1+t+t^{2}\right)$. Since the Hilbert series of the Fomin-Kirillov algebras $\mathcal{E}_{4}$ and $\mathcal{E}_{5}$ have a similar form, we expect that also the latter have a PBW basis.

Corollary 2.14. Any polynomial identity of $\left(\mathbb{K}^{2 \times 2}\right)^{3}$ is a polynomial identity of $\mathcal{D}_{3}\left(\alpha_{1}, \alpha_{2}\right)$. 
Proof. It follows from Theorem 2.11 and an argument similar to Proposition 1.3

The following result classifies PBW deformations of $\mathcal{E}_{3}$. These deformations already appeared in [15, Theorem 6.2] and were used to obtain the classification of finite-dimensional pointed Hopf algebras with coradical isomorphic to $\mathbb{S}_{3}$, see also [2].

Theorem 2.15. Each $P B W$ deformation of $\mathcal{E}_{3}$ in the category of $\mathbb{S}_{3}$-modules is of the form $\mathcal{D}_{3}\left(\gamma_{1}, \gamma_{2}\right), \gamma_{1}, \gamma_{2} \in \mathbb{K}$.

Proof. Let $\mathcal{D}$ be a PBW deformation of $\mathcal{E}_{3}$. Theorem 2.12 for $\alpha_{1}=\alpha_{2}=0$ implies that (2.4) is a basis of $\mathcal{D}$. Since $a^{2}=0$ in $\mathcal{E}_{3}$, there exist $\lambda_{1}, \ldots, \lambda_{4} \in$ $\mathbb{K}$ such that

$$
a^{2}+\lambda_{1} a+\lambda_{2} b+\lambda_{3} c+\lambda_{4}=0
$$

in $\mathcal{D}$. By acting with the transposition (23), see Remark 2.4, one obtains that

$$
2 \lambda_{1} a+\left(\lambda_{2}+\lambda_{3}\right)(b+c)=0
$$

and hence $\lambda_{1}=\lambda_{2}+\lambda_{3}=0$. By acting with (12) and (13) it follows that

$$
b^{2}+\lambda_{2}(c-a)+\lambda_{4}=0, \quad c^{2}+\lambda_{2}(a-b)+\lambda_{4}=0 .
$$

Since $a b+b c+c a=0$ in $\mathcal{E}_{3}$, there exist $\lambda_{5}, \ldots, \lambda_{8} \in \mathbb{K}$ such that

$$
a b+b c+c a+\lambda_{5} a+\lambda_{6} b+\lambda_{7} c+\lambda_{8}=0
$$

in $\mathcal{D}$. By acting with the transpositions (23) and (13) we obtain that

$$
\begin{aligned}
& a c+c b+b a-\lambda_{5} a-\lambda_{6} c-\lambda_{7} b+\lambda_{8}=0, \\
& c b+b a+a c-\lambda_{5} c-\lambda_{6} b-\lambda_{7} a+\lambda_{8}=0 .
\end{aligned}
$$

This implies that $\lambda_{5}=\lambda_{6}=\lambda_{7}$. Now the commutator of $c^{2}+\lambda_{2}(a-b)+\lambda_{4}$ and $c$ in $\mathcal{D}$ becomes $\lambda_{2}(a b-b a)$ up to linear and constant terms and hence $\lambda_{2}=0$. Finally, let $x=a b+b c+c a+\lambda_{5}(a+b+c)+\lambda_{8}$. Then by using other quadratic relations it follows that $x a-b x=a b a-b a b+\lambda_{5}(c a-b c)$ up to linear and constant terms. Let $y=x a-b x$. Then

$$
y-(12) \cdot y=\lambda_{5}(c a-b c-c b+a c)
$$

up to linear and constant terms. Hence $\lambda_{5}=0$ and $\mathcal{D}=\mathcal{D}_{3}\left(-\lambda_{4},-\lambda_{8}\right)$.

We now describe the cases where $\mathcal{D}_{3}\left(\alpha_{1}, \alpha_{2}\right)$ is not semisimple.

Proposition 2.16. Assume that $\alpha_{1} \neq 0$. Then the deformation $\mathcal{D}_{3}\left(\alpha_{1},-\alpha_{1}\right)$ is isomorphic to the product of three copies of the preprojective algebra of the Dynkin quiver of type $A_{2}$.

Proof. It follows directly from Lemma 2.7. 
Proposition 2.17. Assume that $\alpha_{1} \neq 0$. Then the deformation $\mathcal{D}_{3}\left(\alpha_{1}, 3 \alpha_{1}\right)$ can be presented as a quiver with relations in the following way: The quiver has two vertices 1 and 2 , there are two arrows $u_{12}, v_{12}$ from 1 to 2 and two arrows $u_{21}, v_{21}$ from 2 to 1 . The relations are those of the coinvariant ring of $\mathbb{S}_{3}$, i.e.

$$
u_{i j} v_{j i}=v_{i j} u_{j i}, \quad u_{i j} v_{j i}+v_{i j} w_{j i}+u_{i j} w_{j i}=0, \quad u_{i j} v_{j i} w_{i j}=0
$$

for all $i, j \in\{1,2\}$ with $i+j=3$, where $w_{k l}=-u_{k l}-v_{k l}$ for all $k, l \in\{1,2\}$ with $k+l=3$.

Proof. By Theorem [2.12, $\operatorname{dim} \mathcal{D}_{3}\left(\alpha_{1}, \alpha_{2}\right)=12$. Let

$$
f_{1}=\frac{\sqrt{\alpha_{1}}+b}{2 \sqrt{\alpha_{1}}}, \quad f_{2}=\frac{\sqrt{\alpha_{1}}-b}{2 \sqrt{\alpha_{1}}} .
$$

Then $f_{1}$ and $f_{2}$ are primitive idempotents. (The primitivity follows from the fact that the quotient of the deformation by the radical is 2-dimensional, see Proposition 2.10.) The action of the transposition (13) permutes both $f_{1}, f_{2}$ and $u=a-b, v=b-c$. Let $w=-u-v$. By Lemma 2.5, the elements $u$, $v$ and $w$ pairwise commute and

$$
u+v+w=0, \quad u v+v w+u w=0, \quad u v w=0 .
$$

Using the equations

$$
f_{1} u=u f_{2}-\frac{u^{2}}{2 \sqrt{\alpha_{1}}}, \quad f_{2} v=v f_{1}-\frac{v^{2}}{2 \sqrt{\alpha_{1}}}
$$

one can show that the elements $f_{i}, f_{i} u^{2} f_{i}$ and $f_{i} v^{2} f_{i}$ span $f_{i} \mathcal{D}_{3}\left(\alpha_{1}, 3 \alpha_{1}\right) f_{i}$ for $i \in\{1,2\}$, and that $u_{i j}=f_{i} u f_{j}, v_{i j}=f_{i} v f_{j}$ and $f_{i} u^{2} v f_{j}$ for $i, j \in\{1,2\}$ and $i \neq j$ span $f_{i} \mathcal{D}_{3}\left(\alpha_{1}, 3 \alpha_{1}\right) f_{j}$. Then one shows that $f_{i}, u_{i j}$ and $v_{i j}$, where $i, j \in\{1,2\}$ with $i \neq j$, generate the algebra $\mathcal{D}_{3}\left(\alpha_{1}, 3 \alpha_{1}\right)$ and satisfy the relations in the proposition.

\section{An intermediate Algebra}

In this section we assume that $\mathbb{K}$ is an algebraically closed field of characteristic different from 2 and 3. Let $\alpha_{1}, \alpha_{2} \in \mathbb{K}$ and let $\beta=3 \alpha_{1}-\alpha_{2}$.

Definition 3.1. Let $\mathcal{K}\left(\alpha_{1}, \alpha_{2}\right)$ be the associative $\mathbb{K}$-algebra given by generators $a, b, c, y$ and relations

$$
a^{2}=b^{2}=c^{2}=\alpha_{1}, \quad a b+b c+c a=\alpha_{2}, \quad a c+c b+b a=\alpha_{2}+y .
$$

For any $\alpha_{3} \in \mathbb{K}$ let $\mathcal{K}\left(\alpha_{1}, \alpha_{2}, \alpha_{3}\right)=\mathcal{K}\left(\alpha_{1}, \alpha_{2}\right) /\left(y^{3}-\alpha_{3}\right)$.

Lemma 3.2. In the algebra $\mathcal{K}\left(\alpha_{1}, \alpha_{2}\right)$ the following relations hold.

$$
\begin{aligned}
y a & =c y, \quad y b=a y, \quad y c=b y, \\
b a b-a b a & =\alpha_{2}(b-a), \quad(b-a)^{3}=\beta(b-a) .
\end{aligned}
$$

In particular, $y^{3}$ is a central element of $\mathcal{K}\left(\alpha_{1}, \alpha_{2}\right)$. 
Proof. First we obtain that

$$
\begin{aligned}
0 & =b\left(a b+b c+c a-\alpha_{2}\right)-\left(a b+b c+c a-\alpha_{2}\right) a \\
& =b a b+\alpha_{1} c+b c a-\alpha_{2} b-a b a-b c a-\alpha_{1} c+\alpha_{2} a \\
& =b a b-a b a-\alpha_{2}(b-a) .
\end{aligned}
$$

This implies directly the last two equations of the lemma. Now we conclude that

$$
\begin{aligned}
y b-a y & =\left(a c+c b+b a-\alpha_{2}\right) b-a\left(a c+c b+b a-\alpha_{2}\right) \\
& =a c b+\alpha_{1} c+b a b-\alpha_{2} b-\alpha_{1} c-a c b-a b a+\alpha_{2} a \\
& =0 .
\end{aligned}
$$

The other two commutation rules for $y$ follow from this one using that the cyclic group $C_{3}$ acts on $\mathcal{K}\left(\alpha_{1}, \alpha_{2}, \alpha_{3}\right)$ by permuting the generators $a, b, c$ cyclically and by fixing $y$. Then it is also clear that $y^{3}$ is a central element of $\mathcal{K}\left(\alpha_{1}, \alpha_{2}\right)$.

Lemma 3.3. Let $\zeta \in \mathbb{K}$ be such that $\zeta^{2}+\zeta+1=0$ and let

$$
t=a+b+c, \quad v_{+}=a+\zeta b+\zeta^{2} c, \quad v_{-}=a+\zeta^{-1} b+\zeta^{-2} c
$$

be in $\mathcal{K}\left(\alpha_{1}, \alpha_{2}\right)$. Then

$$
\begin{aligned}
y v_{+} & =\zeta v_{+} y, & y v_{-} & =\zeta^{-1} v_{-} y, \\
v_{+} v_{-} & =\beta+\zeta y, & v_{-} v_{+} & =\beta+\zeta^{2} y, \\
t v_{+} & =-v_{+} t-v_{-}^{2}, & t v_{-} & =-v_{-} t-v_{+}^{2} .
\end{aligned}
$$

Proof. The first two equalities follow from Lemma 3.2. The proof of the other formulas is straightforward from the definitions.

Lemma 3.4. Let $\zeta \in \mathbb{K}$ be such that $\zeta^{2}+\zeta+1=0$. Then the elements $v_{+}^{n_{1}} t^{n_{2}} y^{n_{3}}$ and $v_{-}^{n_{1}} t^{n_{2}} y^{n_{3}}$, where $n_{1}, n_{2}, n_{3} \in \mathbb{N}_{0}$, span $\mathcal{K}\left(\alpha_{1}, \alpha_{2}\right)$.

Proof. Clearly, $v_{+}, v_{-}, t$ and $y$ generate $\mathcal{K}\left(\alpha_{1}, \alpha_{2}\right)$. Since $y t=t y$, (3.1) and (3.3) imply that $\mathcal{K}\left(\alpha_{1}, \alpha_{2}\right)$ is spanned by the monomials

$$
v_{s_{1}} v_{s_{2}} \cdots v_{s_{r}} t^{n_{2}} y^{n_{3}}, \quad r, n_{2}, n_{3} \in \mathbb{N}_{0}, \quad s_{1}, \ldots, s_{r} \in\{-,+\} .
$$

Now use (3.2) and (3.1) to conclude the lemma.

Lemma 3.5. Let $\zeta \in \mathbb{K}$ be such that $\zeta^{2}+\zeta+1=0$. Then the following formulas hold in $\mathcal{K}\left(\alpha_{1}, \alpha_{2}\right)$ :

$$
\begin{aligned}
t^{2} & =y+3 \alpha_{1}+2 \alpha_{2}, \\
t v_{+}^{3}+v_{+}^{3} t & =2 y^{2}-2 \beta y-\beta^{2}, \\
v_{+}^{6} & =y^{3}+\beta^{3}, \\
v_{+}^{3} & =v_{-}^{3} .
\end{aligned}
$$


Proof. The first equality follows from the definitions. Using the last two equations in Lemma 3.3 we obtain that

$$
\begin{aligned}
& t v_{+}^{3}+v_{+}^{3} t=t\left(-t v_{-}-v_{-} t\right) v_{+}+v_{+}\left(-t v_{-}-v_{-} t\right) t \\
& =-t^{2} v_{-} v_{+}+\left(v_{+}^{2}+v_{-} t\right) t v_{+}+v_{+} t\left(v_{+}^{2}+t v_{-}\right)-v_{+} v_{-} t^{2} \\
& =-t^{2} v_{-} v_{+}+v_{-} t^{2} v_{+}+v_{+} t^{2} v_{-}-v_{+} v_{-} t^{2}-v_{+} v_{-}^{2} v_{+}
\end{aligned}
$$

Then (3.5) follows from (3.4) and the other equations in Lemma 3.3. Using (3.3) we obtain that

$$
v_{+}^{3}=v_{+}^{2} v_{+}=-t v_{-} v_{+}-v_{i} t v_{+}=-v_{-} v_{+} t-v_{-} t v_{+}=v_{-} v_{-}^{2}=v_{-}^{3} .
$$

Finally,

$$
v_{+}^{6}=v_{+}^{3} v_{-}^{3}=(\beta+\zeta y)\left(\beta+\zeta^{2} y\right)(\beta+y)=y^{3}+\beta^{3}
$$

because of (3.7), (3.1) and (3.2).

Lemma 3.6. Let $\alpha_{3} \in \mathbb{K} \backslash\{0\}$. There exists $\lambda \in \mathbb{K}$ such that $v_{+}+\lambda v_{-}^{2}$ is invertible in $\mathcal{K}\left(\alpha_{1}, \alpha_{2}, \alpha_{3}\right)$.

Proof. Assume first that $\alpha_{3}+\beta^{3} \neq 0$. Then $v_{+}^{6}$ is a non-zero constant by Equation (3.6) of Lemma 3.5 and hence $\lambda=0$ works. Now assume that $\alpha_{3}+\beta^{3}=0$. Let $\zeta \in \mathbb{K}$ such that $\zeta^{2}+\zeta+1=0$. Since $y^{3}=-\beta^{3}$, there exist orthogonal idempotents $e_{0}, e_{1}, e_{2} \in \mathbb{K}[y]$ such that $y e_{i}=-\zeta^{i} \beta e_{i}$ for all $i \in\{0,1,2\}$ and $e_{0}+e_{1}+e_{2}=1$. Let $\lambda \in \mathbb{K} \backslash\{0\}$. Then Lemma 3.5 implies that

$$
\left(v_{+}+\lambda v_{-}^{2}\right)\left(v_{-}-\lambda v_{+}^{2}\right)=\beta+\zeta y-\lambda^{2}\left(\beta+\zeta^{2} y\right)(\beta+y)
$$

Then

$$
e_{i}\left(v_{+}+\lambda v_{-}^{2}\right)\left(v_{-}-\lambda v_{+}^{2}\right)=e_{i} \beta\left(1-\zeta^{i+1}-\lambda^{2} \beta\left(1-\zeta^{i}\right)\left(1-\zeta^{2+i}\right)\right)
$$

is non-zero for all $i \in\{0,1,2\}$. Hence $v_{+}+\lambda v_{-}^{2}$ is invertible.

For the formulation of the next claim we need additional notation. For any $\gamma \in \mathbb{K}$ let $\left(V, q_{\gamma}\right)$ be a two-dimensional quadratic space with basis $x_{1}, x_{2}$ such that

$q_{\gamma}\left(\lambda_{1} x_{1}+\lambda_{2} x_{2}\right)=\left(\gamma+3 \alpha_{1}+2 \alpha_{2}\right) \lambda_{1}^{2}+\left(2 \gamma^{2}-2 \beta \gamma-\beta^{2}\right) \lambda_{1} \lambda_{2}+\left(\gamma^{3}+\beta^{3}\right) \lambda_{2}^{2}$ for all $\lambda_{1}, \lambda_{2} \in \mathbb{K}$. If $\gamma^{3}+\beta^{3} \neq 0$, then let $x^{\prime}, x^{\prime \prime} \in C\left(V, q_{\gamma}\right)$ be the elements

$$
\begin{gathered}
x^{\prime}=-x_{1}-\left(\beta+\zeta^{2} \gamma\right)^{-1} x_{2}, \\
x^{\prime \prime}=x_{1}+\left(\beta+\zeta^{2} \gamma\right)^{-1} x_{2}-(\beta+\gamma)^{-1} x_{2}
\end{gathered}
$$


and let $Y, A, B, C \in C\left(V, q_{\gamma}\right)^{3 \times 3}$ be the matrices

$$
\begin{aligned}
Y & =\left(\begin{array}{ccc}
\gamma & 0 & 0 \\
0 & \zeta \gamma & 0 \\
0 & 0 & \zeta^{2} \gamma
\end{array}\right), \\
A & =\frac{1}{3}\left(\begin{array}{ccc}
x_{1} & \left(\beta+\zeta^{2} \gamma\right) & x_{2} \\
1 & x^{\prime} & \beta+\gamma \\
(\beta+\zeta \gamma) x_{2}^{-1} & 1 & x^{\prime \prime}
\end{array}\right), \\
B & =\frac{1}{3}\left(\begin{array}{ccc}
x_{1} & \zeta\left(\beta+\zeta^{2} \gamma\right) & \zeta^{2} x_{2} \\
\zeta^{2} & x^{\prime} & \zeta(\beta+\gamma) \\
\zeta(\beta+\zeta \gamma) x_{2}^{-1} & \zeta^{2} & x^{\prime \prime}
\end{array}\right) \\
C & =\frac{1}{3}\left(\begin{array}{ccc}
x_{1} & \zeta^{2}\left(\beta+\zeta^{2} \gamma\right) & \zeta x_{2} \\
\zeta & x^{\prime} & \zeta^{2}(\beta+\gamma) \\
\zeta^{2}(\beta+\zeta \gamma) x_{2}^{-1} & \zeta & x^{\prime \prime}
\end{array}\right) .
\end{aligned}
$$

Remark 3.7. The discriminant of the quadratic form $q_{\gamma}$ above is

$$
-9\left(4 \alpha_{1} \gamma^{3}+\beta^{3}\left(\alpha_{1}+\alpha_{2}\right)\right) .
$$

Thus the quadratic space $\left(V, q_{\gamma}\right)$ is nondegenerate if and only if this expression is non-zero.

Lemma 3.8. Let $\gamma, \zeta \in \mathbb{K}$ with $\zeta^{2}+\zeta+1=0$. Assume that $\gamma^{3}+\beta^{3} \neq 0$. Then there exists an algebra map $\rho_{\gamma}: \mathcal{K}\left(\alpha_{1}, \alpha_{2}\right) \rightarrow C\left(V, q_{\gamma}\right)^{3 \times 3}$ such that

$$
\rho_{\gamma}(a)=A, \quad \rho_{\gamma}(b)=B, \quad \rho_{\gamma}(c)=C, \quad \rho_{\gamma}(y)=Y .
$$

This map also satisfies the identity $\rho_{\gamma}\left(y^{3}\right)=\gamma^{3} \mathrm{id}$.

Proof. The matrices $A, B, C, Y$ are well-defined. It is straightforward to check that the equations

$$
\begin{gathered}
A^{2}=B^{2}=C^{2}=\alpha_{1} \mathrm{id}, \\
A B+B C+C A=\alpha_{2} \mathrm{id}, \quad A C+C B+B A=\alpha_{2} \mathrm{id}+Y, \quad Y^{3}=\gamma^{3} \mathrm{id}
\end{gathered}
$$
hold.

Proposition 3.9. Let $\gamma, \zeta \in \mathbb{K}$ such that $\zeta^{2}+\zeta+1=0$ and $\gamma\left(\gamma^{3}+\beta^{3}\right) \neq 0$. Let $M$ be a simple $C\left(V, q_{\gamma}\right)$-module. Then $M^{3}$ is a simple $\mathcal{K}\left(\alpha_{1}, \alpha_{2}, \gamma^{3}\right)$ module via

$$
x m=\rho_{\gamma}(x) m \quad \text { for all } x \in \mathcal{K}\left(\alpha_{1}, \alpha_{2}, \gamma^{3}\right), m \in M^{3} .
$$

Proof. Lemma 3.8 implies that $M^{3}$ is a left $\mathcal{K}\left(\alpha_{1}, \alpha_{2}, \gamma^{3}\right)$-module. Let $N$ be a non-zero submodule of $M^{3}$. Since $\mathbb{K}$ is algebraically closed, there exists an eigenvector $m \in N$ of $\rho_{\gamma}(y)$. Moreover, Equation (3.6) implies that $\rho_{\gamma}\left(v_{+}^{6}\right)=\left(\gamma^{3}+\beta^{3}\right)$ id $\neq 0$. Thus, by (3.1) we may assume that $y m=\gamma m$. Since $\gamma \neq 0$, we conclude that $m \in M \times\{0\} \times\{0\}$. Now observe that

$$
x_{1} m^{\prime}=\rho_{\gamma}(t) m^{\prime}, \quad x_{2} m^{\prime}=\rho_{\gamma}\left(v_{+}^{3}\right) m^{\prime} \quad \text { for all } m^{\prime} \in M \times\{0\} \times\{0\} .
$$

Hence the simplicity of $M$ implies that $M \times\{0\} \times\{0\} \subseteq N$. Then $N=M$ because of (3.1). 
Theorem 3.10. Let $\alpha_{3} \in \mathbb{K}$.

(1) The elements

$$
(a-b)^{n_{1}} a^{n_{2}} c^{n_{3}} y^{n_{4}}, \quad n_{1}, n_{4} \in\{0,1,2\}, n_{2}, n_{3} \in\{0,1\},
$$

form a basis of $\mathcal{K}\left(\alpha_{1}, \alpha_{2}, \alpha_{3}\right)$. In particular, $\operatorname{dim} \mathcal{K}\left(\alpha_{1}, \alpha_{2}, \alpha_{3}\right)=36$.

(2) $\mathcal{K}\left(\alpha_{1}, \alpha_{2}, \alpha_{3}\right)$ is a PBW deformation of $\mathcal{K}(0,0,0)$.

Proof. Similarly to the proof of Theorem 2.12 one shows that the elements in (1) span $\mathcal{K}\left(\alpha_{1}, \alpha_{2}, \alpha_{3}\right)$. In particular, $\operatorname{dim} \mathcal{K}\left(\alpha_{1}, \alpha_{2}, \alpha_{3}\right) \leq 36$.

Let $\gamma \in \mathbb{K}$ with $\gamma^{3}=\alpha_{3}$. Assume that

$$
\gamma\left(\gamma^{3}+\beta^{3}\right)\left(4 \alpha_{1} \gamma^{3}+\beta^{3}\left(\alpha_{1}+\alpha_{2}\right)\right) \neq 0 .
$$

By Remark 3.7, the quadratic space $\left(V, q_{\gamma}\right)$ is nondegenerate. Thus $C\left(V, q_{\gamma}\right)$ is simple by Theorem 1.4(2). Hence there exists a 2-dimensional simple $C\left(V, q_{\gamma}\right)$-module. Since $\gamma\left(\gamma^{3}+\beta^{3}\right) \neq 0$, by Proposition 3.9 there exists a simple 6-dimensional $\mathcal{K}\left(\alpha_{1}, \alpha_{2}, \gamma^{3}\right)$-module. Hence $\operatorname{dim} \mathcal{K}\left(\alpha_{1}, \alpha_{2}, \gamma^{3}\right)=36$. The variety of all triples $\left(\alpha_{1}, \alpha_{2}, \gamma^{3}\right)$ satisfying (3.9) is a dense subvariety of $\mathbb{K}^{3}$. Therefore the theorem follows from Proposition 1.3

In order to determine the radical of the deformation $\mathcal{K}\left(\alpha_{1}, \alpha_{2}, \alpha_{3}\right)$ the following proposition is useful.

Proposition 3.11. Let $A$ be a $\mathbb{K}$-algebra and $x, y \in A$. Let $n \in \mathbb{N}$ and $\zeta, \lambda \in \mathbb{K} \backslash\{0\}$. Assume that char $\mathbb{K}$ does not divide $n, \zeta$ is a primitive root of 1 of order $n, x$ is invertible, $y x=\zeta x y$, and $y^{n}=\lambda$. Then there exists a primitive idempotent $e \in \mathbb{K}[y]$ such that the following claims hold.

(1) $A=\oplus_{i, j=0}^{n-1} x^{i} e A e x^{j}$.

(2) The map given by $I \mapsto e I e$ is a bijection between the ideals of $A$ and the ideals of eAe. The inverse map is given by eIe $\mapsto$ AeIeA.

(3) $\operatorname{Rad} A=\oplus_{i, j=0}^{n-1} x^{i} \operatorname{Rad}(e A e) x^{j}$.

Proof. Let $\gamma \in \mathbb{K}$ be such that $\gamma^{n}=\lambda$. Since char $\mathbb{K} \nmid n$ and $y^{n}=\lambda$, there exist unique idempotents $e=e_{0}, e_{1}, \ldots, e_{n-1} \in \mathbb{K}[y]$ such that $y e_{i}=\gamma \zeta^{i} e_{i}$ for all $i$. Moreover, these idempotents are orthogonal and primitive. Using these properties, one checks that $e_{i}=x^{i+k n} e x^{-i-k n}$ for all $i$ and all $k \in \mathbb{Z}$.

(1) Use that $A=\oplus_{i, j=0}^{n-1} e_{i} A e_{j}$ and that $e_{i}=x^{i} e x^{-i}$ for all $i$.

(2) One has to check that $I=A e I e A$ for all ideals $I$ of $A$ and that $e A e J e A e=e J e$ for all ideals $e J e$ of $e A e$. The second claim is obvious. The first one follows from

$$
I=\sum_{i, j=0}^{n-1} e_{i} I e_{j}=\sum_{i, j=0}^{n-1} x^{i} e x^{-i} I x^{j} e x^{-j} \subseteq \text { AeIeA. }
$$

(3) Since $\operatorname{Rad}(e A e)=e(\operatorname{Rad} A) e,(2)$ implies that

$$
\operatorname{Rad} A=A e(\operatorname{Rad} A) e A=\bigoplus_{i, j=0}^{n-1} e_{i} A e \operatorname{Rad}(e A e) e A e_{j} .
$$


Now use that $e_{i}=x^{i} e x^{-i}$ and $e_{j}=x^{j-n} e x^{n-j}$ for all $i, j \in\{1, \ldots, n-1\}$ and that $\operatorname{Rad}(e A e)$ is an ideal of $e A e$.

Lemma 3.12. Let $\gamma \in \mathbb{K} \backslash\{0\}$ and $e \in \mathbb{K}[y] \subseteq \mathcal{K}\left(\alpha_{1}, \alpha_{2}, \gamma^{3}\right)$ be an idempotent. Assume that ye $=\gamma$ e. Then the algebra eK $\left(\alpha_{1}, \alpha_{2}, \gamma^{3}\right)$ e is generated by et and $e v_{+}^{3}$ and is isomorphic to the Clifford algebra $C\left(V, q_{\gamma}\right)$.

Proof. Let $A=\mathcal{K}\left(\alpha_{1}, \alpha_{2}, \gamma^{3}\right)$ and let $\zeta \in \mathbb{K}$ be such that $\zeta^{2}+\zeta+1=0$. Then $A$ is generated by $t, v_{+}, v_{-}$and $y$. Lemma 3.3 implies that $v_{+}^{n_{1}} t^{n_{2}} y^{n_{3}}$, $v_{-}^{n_{1}} t^{n_{2}} y^{n_{3}}$, where $n_{1}, n_{2}, n_{3} \in \mathbb{N}_{0}$, span $A$.

By Lemma 3.6. there exists $\lambda \in \mathbb{K}$ such that $x=v_{+}+\lambda v_{-}^{2} \in A$ is invertible. Moreover, $y x=\zeta x y$ by Lemma 3.3. Then Proposition 3.11 implies that $A=\oplus_{i, j=0}^{2} x^{i} e A e x^{j}$. Note that $v_{+}^{n_{1}} t^{n_{2}} y^{n_{3}} e \in x^{n_{1}^{\prime}} e A e$ and $v_{-}^{m_{1}} t^{n_{2}} y^{n_{3}} e \in x^{m_{1}^{\prime}} e A e$ for all $n_{1}, m_{1}, n_{2}, n_{3} \in \mathbb{N}_{0}$, where $n_{1}^{\prime}, m_{1}^{\prime} \in\{0,1,2\}$ such that $n_{1} \equiv n_{1}^{\prime}(\bmod 3)$ and $m_{1} \equiv-m_{1}^{\prime}(\bmod 3)$. Therefore $e A e$ is generated by $e y=\gamma e$, et and $e v_{+}^{3}=e v_{-}^{3}$, see (3.7). Moreover, Theorem 3.10(1) and Proposition [3.11(1) imply that $\operatorname{dim} e A e=4$. Then the claim follows from Lemma 3.5.

Corollary 3.13. Let $\gamma \in \mathbb{K}$.

(1) If $\gamma=0$ then $y$ generates a nilpotent ideal of $\mathcal{K}\left(\alpha_{1}, \alpha_{2}, \gamma^{3}\right)$.

(2) If $\gamma \neq 0$ then $\mathcal{K}\left(\alpha_{1}, \alpha_{2}, \gamma^{3}\right)$ is semisimple if and only if $q_{\gamma}$ is nondegenerate. In this case, $\mathcal{K}\left(\alpha_{1}, \alpha_{2}, \gamma^{3}\right) \simeq \mathbb{K}^{6 \times 6}$.

Proof. Let $A=\mathcal{K}\left(\alpha_{1}, \alpha_{2}, \gamma^{3}\right)$.

(1) Lemma 3.2 implies that $N=A y$ is a two-sided ideal of $A$. If $\gamma=0$, then $y^{3}=0$ in $A$ and hence $N^{3}=0$.

(2) Assume that $\gamma \neq 0$. Let $\zeta \in \mathbb{K}$ be such that $\zeta^{2}+\zeta+1=0$. By Lemma 3.6, there exists an invertible element $x \in A$ such that $y x=\zeta x y$. According to Proposition 3.11, there exists a primitive idempotent $e \in \mathbb{K}[y]$ such that $\operatorname{Rad} A=\oplus_{i, j=0}^{2} x^{i} \operatorname{Rad}(e A e) x^{j}$. Let $\gamma \in \mathbb{K} \backslash\{0\}$ be such that $y e=\gamma e$. Then $e A e \simeq C\left(V, q_{\gamma}\right)$ by Lemma 3.12. Thus the claim on the semisimplicity of $A$ follows from Theorem 1.4

Assume that $q_{\gamma}$ is nondegenerate. Then $C\left(V, q_{\gamma}\right)$ is simple by Theorem 1.4(2). Hence $A$ is simple by Proposition 3.11 (2). Since $\operatorname{dim} A=36$ by Theorem 3.10(1), we conclude that $A \simeq \mathbb{K}^{6 \times 6}$.

\section{The Nichols Algebra of Dimension 72}

Again we assume that $\mathbb{K}$ is an algebraically closed field of characteristic different from 2 and 3 . In this section we study the algebra $B$ presented by generators $a, b, c, d$ and relations

$$
\begin{aligned}
& a^{2}=b^{2}=c^{2}=d^{2}=0, \\
& a b+b c+c a=a c+c d+d a=a d+b a+d b=b d+c b+d c=0, \\
& (a+b+c)^{6}=0 .
\end{aligned}
$$


Based on computer calculations it is known that $\operatorname{dim} B=72$ and that the Hilbert series of $B$ is

$$
H(t)=1+4 t+8 t^{2}+11 t^{3}+12 t^{4}+12 t^{5}+11 t^{6}+8 t^{7}+4 t^{8}+t^{9},
$$

see [16]. In Theorem 4.9 we will prove these facts by different methods.

Definition 4.1. For any $\alpha_{1}, \alpha_{2} \in \mathbb{K}$, let $\mathcal{T}\left(\alpha_{1}, \alpha_{2}\right)$ be the $\mathbb{K}$-algebra given by generators $a, b, c, d$ and relations

$$
\begin{aligned}
& a^{2}-\alpha_{1}=b^{2}-\alpha_{1}=c^{2}-\alpha_{1}=d^{2}-\alpha_{1}=0, \\
& c a+b c+a b-\alpha_{2}=d a+c d+a c-\alpha_{2}=0, \\
& d b+b a+a d-\alpha_{2}=d c+c b+b d-\alpha_{2}=0 .
\end{aligned}
$$

Let $y=a c+c b+b a-\alpha_{2}$.

Remark 4.2. Let $G$ be the group given by generators $g_{a}, g_{b}, g_{c}, g_{d}$ with relations

$$
\begin{array}{ll}
g_{a} g_{b}=g_{b} g_{c}=g_{c} g_{a}, & g_{a} g_{c}=g_{c} g_{d}=g_{d} g_{a}, \\
g_{a} g_{d}=g_{d} g_{b}=g_{b} g_{a}, & g_{b} g_{d}=g_{d} g_{c}=g_{c} g_{b} .
\end{array}
$$

It is known that $G$ is a central extension of $\operatorname{SL}(2,3)$, see [17].

There is a unique $\mathbb{K} G$-module algebra structure on $\mathcal{T}\left(\alpha_{1}, \alpha_{2}\right)$ such that $g_{a}, g_{b}, g_{c}, g_{d}$ act on the generators $a, b, c, d$ according to Table 4.1 .

TABLE 4.1. The action of $G$.

\begin{tabular}{c|cccc} 
& $a$ & $b$ & $c$ & $d$ \\
\hline$g_{a}$ & $-a$ & $-c$ & $-d$ & $-b$ \\
$g_{b}$ & $-d$ & $-b$ & $-a$ & $-c$ \\
$g_{c}$ & $-b$ & $-d$ & $-c$ & $-a$ \\
$g_{d}$ & $-c$ & $-a$ & $-b$ & $-d$
\end{tabular}

Remark 4.3. The usual presentation for $B$ found in the literature involves a different degree-six relation. One computes

$$
(c b)^{2}=c(b c) b=c(-a b-c a) b=0 .
$$

Then acting on this with $g_{d}$ and $g_{d}^{2}$ one obtains $(a c)^{2}=(b a)^{2}=0$. Now a direct computation shows that

$$
(c b+b a+a c)^{3}=(c b a)^{2}+(b a c)^{2}+(a c b)^{2} .
$$

Remark 4.4. The algebra $B$ together with an appropriate comultiplication, counit and antipode is the Nichols algebra associated with the rack given by a conjugacy class of 3 -cycles in the alternating group $\mathbb{A}_{4}$ and constant cocycle -1. It was found by Graña in [16] and later used by Ngakeu, Majid and Lambert in noncommutative geometry [25]. The group $G$ of Remark 4.2 is the enveloping group of this rack. 
Lemma 4.5. Let $\alpha_{1}, \alpha_{2} \in \mathbb{K}$. Then $y x=-\left(g_{d} \cdot x\right) y$ in $\mathcal{T}\left(\alpha_{1}, \alpha_{2}\right)$ for all $x \in\{a, b, c, d\}$.

Proof. Let $X=\{a, b, c, d\}$. The element $g_{d}^{2} \in G$, where $G$ is the group in Remark 4.2, permutes the elements $b, c, d$ cyclically and fixes $y$. Therefore it suffices to prove that

$$
y d=d y, \quad y b=a y .
$$

Equation $y d=d y$ is proved as follows.

$$
\begin{aligned}
y d & =\left(b a+a c+c b-\alpha_{2}\right) d \\
& =b\left(\alpha_{2}-d b-b a\right)+a\left(\alpha_{2}-d a-a c\right)+c\left(\alpha_{2}-d c-c b\right)-\alpha_{2} d \\
& =\left(\alpha_{2}-b d\right) b+\left(\alpha_{2}-a d\right) a+\left(\alpha_{2}-c d\right) c-\alpha_{2} d-\alpha_{1}(a+b+c) \\
& =(d c+c b) b+(d b+b a) a+(d a+a c) c-\alpha_{2} d-\alpha_{1}(a+b+c) \\
& =d y .
\end{aligned}
$$

Equation $y b=c y$ is obtained by the following steps:

$$
\begin{aligned}
y b & =\left(b a+a c+c b-\alpha_{2}\right) b \\
& =b\left(a b-\alpha_{2}\right)+a c b+\alpha_{1} c \\
& =b(-c a-b c)+a(c b+a c) \\
& =-\alpha_{1} c-\left(\alpha_{2}-c a-a b\right) a+a(c b+a c) \\
& =a\left(-\alpha_{2}+b a+c b+a c\right) \\
& =a y .
\end{aligned}
$$

This completes the proof.

Definition 4.6. For any $\alpha_{1}, \alpha_{2}, \alpha_{3} \in \mathbb{K}$, let $\mathcal{T}\left(\alpha_{1}, \alpha_{2}, \alpha_{3}\right)$ be the deformation of $B$ given by generators $a, b, c, d$ and relations

$$
\begin{aligned}
& a^{2}-\alpha_{1}=b^{2}-\alpha_{1}=c^{2}-\alpha_{1}=d^{2}-\alpha_{1}=0, \\
& c a+b c+a b-\alpha_{2}=d a+c d+a c-\alpha_{2}=0, \\
& d b+b a+a d-\alpha_{2}=d c+c b+b d-\alpha_{2}=0, \\
& \left(c b+b a+a c-\alpha_{2}\right)^{3}-\alpha_{3}=0 .
\end{aligned}
$$

Remark 4.7. Let $\alpha_{1}, \alpha_{2}, \alpha_{3} \in \mathbb{K}$. The algebra $\mathcal{T}\left(\alpha_{1}, \alpha_{2}, \alpha_{3}\right)$ is naturally a $\mathcal{K}\left(\alpha_{1}, \alpha_{2}, \alpha_{3}\right)$-bimodule where the action of $a, b, c$ and $y$ is given by multiplication with $a, b, c$ and $y$, respectively.

Proposition 4.8. For any $\alpha_{1}, \alpha_{2}, \alpha_{3} \in \mathbb{K}$, the algebra $\mathcal{T}\left(\alpha_{1}, \alpha_{2}, \alpha_{3}\right)$ is isomorphic to the Ore extension $\mathcal{K}\left(\alpha_{1}, \alpha_{2}, \alpha_{3}\right)[d ; \partial, \sigma] /\left(d^{2}-\alpha_{1}\right)$, where

$$
\sigma \in \operatorname{Aut}\left(\mathcal{K}\left(\alpha_{1}, \alpha_{2}, \alpha_{3}\right)\right), \quad \sigma(a)=-c, \quad \sigma(b)=-a, \quad \sigma(c)=-b,
$$

and the $(\sigma, \mathrm{id})$-skew derivation $\partial$ of $\mathcal{K}\left(\alpha_{1}, \alpha_{2}, \alpha_{3}\right)$ is given by

$$
\partial(a)=\alpha_{2}-a c, \quad \partial(b)=\alpha_{2}-b a, \quad \partial(c)=\alpha_{2}-c b .
$$


Proof. It is straightforward to prove that the automorphism $\sigma$ and the skew derivation $\partial$ of $\mathcal{K}\left(\alpha_{1}, \alpha_{2}, \alpha_{3}\right)$ exist. For example:

$$
\partial\left(a^{2}\right)=\partial(a) a+\sigma(a) \partial(a)=\left(\alpha_{2}-a c\right) a-c\left(\alpha_{2}-a c\right)=0 .
$$

The rest follows from the definitions of $\mathcal{K}\left(\alpha_{1}, \alpha_{2}, \alpha_{3}\right)$ and $\mathcal{T}\left(\alpha_{1}, \alpha_{2}, \alpha_{3}\right)$.

Recall from Definition 4.1 that $y=a c+c b+b a-\alpha_{2}$.

Theorem 4.9. For any $\alpha_{1}, \alpha_{2}, \alpha_{3} \in \mathbb{K}$ the algebra $\mathcal{T}\left(\alpha_{1}, \alpha_{2}, \alpha_{3}\right)$ is a PBW deformation of $B$ and

$$
(b-a)^{n_{1}} a^{n_{2}} c^{n_{3}} y^{n_{4}} d^{n_{5}}, \quad n_{1}, n_{4} \in\{0,1,2\}, n_{2}, n_{3}, n_{5} \in\{0,1\},
$$

is a basis of $\mathcal{T}\left(\alpha_{1}, \alpha_{2}, \alpha_{3}\right)$.

Proof. First one checks that $\left(d^{2}-\alpha_{1}\right) a=b\left(d^{2}-\alpha_{1}\right)$. By acting on this equation with $g_{d} \in G$ it follows that the left ideal of $\mathcal{T}\left(\alpha_{1}, \alpha_{2}, \alpha_{3}\right)$ generated by $d^{2}-\alpha_{1}$ is a two-sided ideal. Hence

$$
\mathcal{T}\left(\alpha_{1}, \alpha_{2}, \alpha_{3}\right) \simeq \mathcal{K}\left(\alpha_{1}, \alpha_{2}, \alpha_{3}\right) \otimes \mathbb{K}[d] /\left(d^{2}-\alpha_{1}\right)
$$

as a left module over $\mathcal{K}\left(\alpha_{1}, \alpha_{2}, \alpha_{3}\right)$ by Proposition 4.8, see Remark 4.7 Now apply Theorem 3.10(1) to obtain the claimed basis of $\mathcal{T}\left(\alpha_{1}, \alpha_{2}, \alpha_{3}\right)$. Hence $\operatorname{dim} \mathcal{T}\left(\alpha_{1}, \alpha_{2}, \alpha_{3}\right)=\operatorname{dim} B=72$ for all $\alpha_{1}, \alpha_{2}, \alpha_{3} \in \mathbb{K}$. Therefore $\mathcal{T}\left(\alpha_{1}, \alpha_{2}, \alpha_{3}\right)$ is a PBW deformation of $B$.

Remark 4.10. In view of Theorem 4.9 it is reasonable to ask for the defining relations of $\mathcal{T}\left(\alpha_{1}, \alpha_{2}, \alpha_{3}\right)$ in terms of the generators $b-a, a, c, y, d$. By translating the nine defining relations in Definition 4.6 one obtains the relations

$$
\begin{aligned}
a^{2} & =c^{2}=d^{2}=\alpha_{1}, \\
a(b-a) & =-(b-a) a-(b-a)^{2}, \\
c a & =-a c-(b-a) c+(b-a) a+(b-a)^{2}+\alpha_{2}-\alpha_{1}, \\
d a & =-a c-c d+\alpha_{2}, \\
d(b-a) & =c d-a d+a c-(b-a) a-\alpha_{1}, \\
y & =c(b-a)-(b-a) c+2(b-a) a+(b-a)^{2}, \\
y^{3} & =\alpha_{1}
\end{aligned}
$$

(which is not something we prefer to work with). Nevertheless, the theorem implies directly that the Hilbert series of $B$ is the polynomial

$$
H(t)=(1+t)^{3}\left(1+t+t^{2}\right)\left(1+t^{2}+t^{4}\right) .
$$

Theorem 4.11. The algebra $\mathcal{T}\left(\alpha_{1}, \alpha_{2}, \alpha_{3}\right)$ is semisimple if and only if

$$
\alpha_{3}\left(\alpha_{3}+\left(\alpha_{1}+\alpha_{2}\right)\left(3 \alpha_{1}-\alpha_{2}\right)^{2}\right) \neq 0 .
$$

In this case $\mathcal{T}\left(\alpha_{1}, \alpha_{2}, \alpha_{3}\right) \simeq\left(\mathbb{K}^{6 \times 6}\right)^{2}$. 
Proof. Assume that $\alpha_{3}=0$. Lemma 4.5 implies that the left ideal generated by $y$ is a two-sided nilpotent ideal. Hence $\mathcal{T}\left(\alpha_{1}, \alpha_{2}, 0\right)$ is not semisimple.

Now assume that $\alpha_{3} \neq 0$. Let $\zeta \in \mathbb{K}$ be such that $\zeta^{2}+\zeta+1=0$. Lemma 3.6 and (3.1) imply that there exists an invertible element $x \in$ $\mathcal{T}\left(\alpha_{1}, \alpha_{2}, \alpha_{3}\right)$ such that $y x=\zeta x y$. By Proposition 3.11(3), there exists a primitive idempotent $e \in \mathbb{K}[y]$ such that $\mathcal{T}\left(\alpha_{1}, \alpha_{2}, \alpha_{3}\right)$ is semisimple if and only if $T=e \mathcal{T}\left(\alpha_{1}, \alpha_{2}, \alpha_{3}\right) e$ is semisimple. Since $y^{3}=\alpha_{3}$, there exists $\gamma \in \mathbb{K}$ such that $y e=\gamma e$ and $\gamma^{3}=\alpha_{3}$.

Recall that $y d=d y$ by Lemma 4.5. Therefore $T$ is generated by et, $e v_{+}^{3}$ and $e d$ by (4.1) and Lemma 3.12, where $t=a+b+c$ and $v_{+}=a+\zeta b+\zeta^{2} c$. Moreover, $\operatorname{dim} T=8$. Now we obtain that

$$
d^{2}=\alpha_{1}, \quad d t+t d=2 \alpha_{2}-y, \quad d v_{+}^{3}+v_{+}^{3} d=2 y^{2}+\left(3 \alpha_{1}-\alpha_{2}\right)^{2} .
$$

Hence $T$ is isomorphic to the Clifford algebra $C\left(W, q_{\gamma}^{\prime}\right)$, where $W$ is a threedimensional vector space and $q_{\gamma}^{\prime}$ is the quadratic form on $W$ given by

$$
\begin{aligned}
q_{\gamma}^{\prime}\left(\lambda_{1} x_{1}\right. & \left.+\lambda_{2} x_{2}+\lambda_{3} x_{3}\right)=\left(\gamma+3 \alpha_{1}+2 \alpha_{2}\right) \lambda_{1}^{2}+\left(\gamma^{3}+\beta^{3}\right) \lambda_{2}^{2}+\alpha_{1} \lambda_{3}^{2} \\
& +\left(2 \gamma^{2}-2 \beta \gamma-\beta^{2}\right) \lambda_{1} \lambda_{2}+\left(2 \alpha_{2}-\gamma\right) \lambda_{1} \lambda_{3}+\left(2 \gamma^{2}+\beta^{2}\right) \lambda_{2} \lambda_{3}
\end{aligned}
$$

with respect to a fixed basis $x_{1}, x_{2}, x_{3}$ of $W$, and $\beta=3 \alpha_{1}-\alpha_{2}$. The semisimplicity of $C\left(W, q_{\gamma}^{\prime}\right)$ is equivalent to the nondegeneracy of $q_{\gamma}^{\prime}$, that is, to $\alpha_{3}+\left(\alpha_{1}+\alpha_{2}\right)\left(3 \alpha_{1}-\alpha_{2}\right)^{2} \neq 0$.

Assume now that $\mathcal{T}\left(\alpha_{1}, \alpha_{2}, \alpha_{3}\right)$ (equivalently, $\left.T\right)$ is semisimple. By Proposition 3.11 (2) and Theorem 1.4 (3), the algebra $\mathcal{T}\left(\alpha_{1}, \alpha_{2}, \alpha_{3}\right)$ is the direct product of two simple ideals. Then the claim follows from the fact that $72=6^{2}+6^{2}$ is the only decomposition of 72 as a sum of two squares.

A result analogous to Corollary 2.14 is the following:

Corollary 4.12. Any polynomial identity of $\left(\mathbb{K}^{6 \times 6}\right)^{2}$ is a polynomial identity of $\mathcal{T}\left(\alpha_{1}, \alpha_{2}, \alpha_{3}\right)$ for any $\alpha_{1}, \alpha_{2}, \alpha_{3} \in \mathbb{K}$.

Recall the definition of the group $G$ in Remark 4.2. The following result classifies PBW deformation of the algebra $B$. These deformations already appeared in [15, Theorem 6.3] in the context of the classification of finitedimensional pointed Hopf algebras with non-abelian coradical.

Theorem 4.13. Each $P B W$ deformation of $B$ in the category of $G$-modules is of the form $\mathcal{T}\left(\alpha_{1}, \alpha_{2}, \alpha_{3}\right), \alpha_{1}, \alpha_{2}, \alpha_{3} \in \mathbb{K}$.

Proof. Let $T$ be a PBW deformation of $B$ in the category of $G$-modules. Theorem 4.9 for $B$ implies that $T$ is given by generators $a, b, c, d$ and relations

$$
\begin{gathered}
a^{2}=t_{1}, \quad b^{2}=t_{2}, \quad c^{2}=t_{3}, \quad d^{2}=t_{4}, \\
c a+b c+a b=t_{5}, \quad d a+c d+a c=t_{6}, \\
d b+b a+a d=t_{7}, \quad d c+c b+b d=t_{8}, \\
(c b+b a+a c)^{3}=t_{9},
\end{gathered}
$$


where $t_{1}, t_{2}, \ldots, t_{8}$ are linear combinations of $1, a, b, c, d$, and $t_{9}$ is a linear combination of monomials of degree at most 5 in the generators $a, b, c, d$. By acting with $g_{a}^{3}$ on the equation $a^{2}=t_{1}$ one obtains that $a^{2}=\alpha_{1}$ for some $\alpha \in \mathbb{K}$. By acting with $g_{b}$ and $g_{c}$ we conclude that $b^{2}=c^{2}=d^{2}=\alpha_{1}$. Similarly, the actions of $g_{d}^{3}$ and $g_{b}, g_{c}$ on the equation $c a+b c+a b=t_{5}$ imply that $t_{5}=t_{6}=t_{7}=t_{8}=\alpha_{2}$ for some $\alpha_{2} \in \mathbb{K}$. Let us rewrite the last defining relation of $T$ to be

$$
y^{3}=t_{9}^{\prime},
$$

where $y=c b+b a+a c-\alpha_{2}$. It remains to show that $t_{9}^{\prime} \in \mathbb{K} 1$.

Lemma 3.4 and (4.1) imply that

$$
t_{9}^{\prime}=\sum_{n_{1}, \ldots, n_{4} \in \mathbb{N}_{0}} \lambda_{n_{1}, \ldots, n_{4}} v_{+}^{n_{1}} t^{n_{2}} y^{n_{3}} d^{n_{4}}+\sum_{n_{1}, \ldots, n_{4} \in \mathbb{N}_{0}} \mu_{n_{1}, \ldots, n_{4}} v_{-}^{n_{1}} t^{n_{2}} y^{n_{3}} d^{n_{4}}
$$

for some $\lambda_{n_{1}, \ldots, n_{4}}, \mu_{n_{1}, \ldots, n_{4}} \in \mathbb{K}$. Since $g_{d} \cdot y^{3}=y^{3}$ and

$$
\begin{aligned}
& g_{d} \cdot v_{+}^{n_{1}} t^{n_{2}} y^{n_{3}} d^{n_{4}}=\zeta^{n_{1}}(-1)^{n_{1}+n_{2}+n_{4}} v_{+}^{n_{1}} t^{n_{2}} y^{n_{3}} d^{n_{4}}, \\
& g_{d} \cdot v_{-}^{n_{1}} t^{n_{2}} y^{n_{3}} d^{n_{4}}=\zeta^{-n_{1}}(-1)^{n_{1}+n_{2}+n_{4}} v_{-}^{n_{1}} t^{n_{2}} y^{n_{3}} d^{n_{4}},
\end{aligned}
$$

we conclude that $\lambda_{n_{1}, \ldots, n_{4}}=\mu_{n_{1}, \ldots, n_{4}}=0$ whenever $3 \nmid n_{1}$ or $n_{1}+n_{2}+n_{4}$ is odd. Moreover the degree of $t_{9}^{\prime}$ is at most five. Hence $d^{2}=\alpha_{1}$ and (3.4) imply that

$$
t_{9}^{\prime}=\lambda_{1}+\lambda_{2} t d+\lambda_{3} y+\lambda_{4} v_{+}^{3} t+\lambda_{5} v_{+}^{3} d+\lambda_{6} t y d+\lambda_{7} y^{2}
$$

for some $\lambda_{1}, \ldots, \lambda_{7} \in \mathbb{K}$. Equations $d t=-t d+2 \alpha_{2}-y$ and (3.5) imply that

$$
t_{9}^{\prime} t-t t_{9}^{\prime}=\left(\left(\beta^{2} \lambda_{5}-2 \lambda_{2} t^{2}\right)+2\left(\beta \lambda_{5}-\lambda_{6} t^{2}\right) y-2 \lambda_{5} y^{2}\right) d+t^{\prime \prime},
$$

for some $t^{\prime \prime} \in \mathcal{K}\left(\alpha_{1}, \alpha_{2}\right)$. Since $y^{3} t=t y^{3}$, using (4.1) and Theorem 4.9 we conclude that $\lambda_{5}=0$. Similarly, using (4.2) and $d y=y d$, we obtain that

$$
t_{9}^{\prime} d-d t_{9}^{\prime}=\left(\lambda_{6} y^{2}+\left(\lambda_{2}-2 \alpha_{2} \lambda_{6}\right) y-2 \alpha_{2} \lambda_{2}\right) d+t^{\prime \prime \prime}
$$

for some $t^{\prime \prime \prime} \in \mathcal{K}\left(\alpha_{1}, \alpha_{2}\right)$. Since $y^{3} d=d y^{3}$, we conclude from Theorem 4.9 that $\lambda_{2}=\lambda_{6}=0$. Since

$$
0=t_{9}^{\prime}(t+2 d)-(t+2 d) t_{9}^{\prime}=-6 \lambda_{4} t y^{2}+\text { terms of degree } \leq 3,
$$

Theorem 4.9 implies that $\lambda_{4}=0$. Finally $y^{3} a=a y^{3}$ and Lemma 3.2 imply that

$$
0=t_{9}^{\prime} a-a t_{9}^{\prime}=\lambda_{3}(c-a) y+\lambda_{7}(b-a) y^{2}
$$

and hence $t_{9}^{\prime}=\lambda_{1}$ by Theorem 4.9 .

\section{REFERENCES}

[1] N. Andruskiewitsch, F. Fantino, G. A. García, and L. Vendramin. On Nichols algebras associated to simple racks. In Groups, algebras and applications, volume 537 of Contemp. Math., pages 31-56. Amer. Math. Soc., Providence, RI, 2011.

[2] N. Andruskiewitsch, I. Heckenberger, and H.-J. Schneider. The Nichols algebra of a semisimple Yetter-Drinfeld module. Amer. J. Math., 132(6):1493-1547, 2010. 
[3] N. Andruskiewitsch and H.-J. Schneider. Pointed Hopf algebras. In New directions in Hopf algebras, volume 43 of Math. Sci. Res. Inst. Publ., pages 1-68. Cambridge Univ. Press, Cambridge, 2002.

[4] Y. Bazlov. Nichols-Woronowicz algebra model for Schubert calculus on Coxeter groups. J. Algebra, 297(2):372-399, 2006.

[5] R. Berger and V. Ginzburg. Higher symplectic reflection algebras and nonhomogeneous N-Koszul property. J. Algebra, 304(1):577-601, 2006.

[6] J. Blasiak, R. I. Liu, and K. Mészáros. Subalgebras of the Fomin-Kirillov algebra. J. Algebraic Combin., 44(3):785-829, 2016.

[7] W. Crawley-Boevey and M. P. Holland. Noncommutative deformations of Kleinian singularities. Duke Math. J., 92(3):605-635, 1998.

[8] D. Ştefan and C. Vay. The cohomology ring of the 12-dimensional Fomin-Kirillov algebra. Adv. Math., 291:584-620, 2016.

[9] P. Etingof and V. Ginzburg. Symplectic reflection algebras, Calogero-Moser space, and deformed Harish-Chandra homomorphism. Invent. Math., 147(2):243-348, 2002.

[10] P. Etingof and E. Rains. New deformations of group algebras of Coxeter groups. Int. Math. Res. Not., (10):635-646, 2005.

[11] P. Etingof and E. Rains. New deformations of group algebras of Coxeter groups. II. Geom. Funct. Anal., 17(6):1851-1871, 2008.

[12] G. Fløystad and J. E. Vatne. PBW-deformations of N-Koszul algebras. J. Algebra, 302(1):116-155, 2006.

[13] S. Fomin and A. N. Kirillov. Quadratic algebras, Dunkl elements, and Schubert calculus. In Advances in geometry, volume 172 of Progr. Math., pages 147-182. Birkhäuser Boston, Boston, MA, 1999.

[14] W. L. Gan and V. Ginzburg. Deformed preprojective algebras and symplectic reflection algebras for wreath products. J. Algebra, 283(1):350-363, 2005.

[15] A. García Iglesias and C. Vay. Finite-dimensional pointed or copointed Hopf algebras over affine racks. J. Algebra, 397:379-406, 2014.

[16] M. Graña. On Nichols algebras of low dimension. In New trends in Hopf algebra theory (La Falda, 1999), volume 267 of Contemp. Math., pages 111-134. Amer. Math. Soc., Providence, RI, 2000.

[17] I. Heckenberger and L. Vendramin. Nichols algebras over groups with finite root system of rank two II. J. Group Theory, 17(6):1009-1034, 2014.

[18] S. Lentner. Quantum groups and Nichols algebras acting on conformal field theories. arXiv:1702.06431, 2017.

[19] R. I. Liu. On the commutative quotient of Fomin-Kirillov algebras. European J. Combin., 54:65-75, 2016.

[20] S. Majid. Noncommutative differentials and Yang-Mills on permutation groups $S_{n}$. In Hopf algebras in noncommutative geometry and physics, volume 239 of Lecture Notes in Pure and Appl. Math., pages 189-213. Dekker, New York, 2005.

[21] S. Majid and E. Raineri. Electromagnetism and gauge theory on the permutation group $S_{3}$. J. Geom. Phys., 44(2-3):129-155, 2002.

[22] K. Mészáros, G. Panova, and A. Postnikov. Schur times Schubert via the FominKirillov algebra. Electron. J. Combin., 21(1):Paper 1.39, 22, 2014.

[23] A. Milinski and H.-J. Schneider. Pointed indecomposable Hopf algebras over Coxeter groups. In New trends in Hopf algebra theory (La Falda, 1999), volume 267 of Contemp. Math., pages 215-236. Amer. Math. Soc., Providence, RI, 2000.

[24] T. Mora. An introduction to commutative and noncommutative Gröbner bases. Theoret. Comput. Sci., 134(1):131-173, 1994. Second International Colloquium on Words, Languages and Combinatorics (Kyoto, 1992).

[25] F. Ngakeu, S. Majid, and D. Lambert. Noncommutative Riemannian geometry of the alternating group $A_{4}$. J. Geom. Phys., 42(3):259-282, 2002. 
[26] A. Polishchuk and L. Positselski. Quadratic algebras, volume 37 of University Lecture Series. American Mathematical Society, Providence, RI, 2005.

[27] B. Roehrig. Deformed Fomin-Kirillov Algebras and Applications. PhD thesis, Philipps-Universität, Marburg, 2016. DOI : 10.17192/z2017.0049.

[28] W. Scharlau. Quadratic and Hermitian forms, volume 270 of Grundlehren der Mathematischen Wissenschaften [Fundamental Principles of Mathematical Sciences]. Springer-Verlag, Berlin, 1985.

[29] A. V. Shepler and S. Witherspoon. PBW deformations of skew group algebras in positive characteristic. Algebr. Represent. Theory, 18(1):257-280, 2015.

[30] C. Walton and S. Witherspoon. PBW deformations of braided products. J. Algebra, 504:536-567, 2018.

I. Heckenberger: Philipps-Universität Marburg, FB Mathematik und Informatik, Hans-Meerwein-Strasse, 35032 Marburg, Germany.

E-mail address: heckenberger@mathematik.uni-marburg.de

L. Vendramin: IMAS-COniCET and Depto. de Matemática, FCEN, Universidad de Buenos Aires, Pabellón 1, Ciudad Universitaria (1428), Buenos Aires, Argentina.

E-mail address: lvendramin@dm.uba.ar 